\title{
La fabrique du retour en Afrique. Politiques et pratiques de l'appartenance en Jamaïque (1920-1968)
}

Constructing the Return to Africa. Politics and Practices of Belonging in Jamaica (1920-1968)

La fábrica del retorno en África. Políticas y prácticas de la pertenencia en Jamaica (1920-1968)

\section{Giulia Bonacci}

\section{(2) OpenEdition}

\section{Journals}

Édition électronique

URL : https://journals.openedition.org/remi/6499

DOI : 10.4000/remi.6499

ISSN : $1777-5418$

Éditeur

Université de Poitiers

Édition imprimée

Date de publication : 1 septembre 2013

Pagination : 33-54

ISBN : 979-10-90426-09-2

ISSN : 0765-0752

\section{Référence électronique}

Giulia Bonacci, « La fabrique du retour en Afrique. Politiques et pratiques de l'appartenance en

Jamaïque (1920-1968) », Revue européenne des migrations internationales [En ligne], vol. 29 - n³ | 2013, mis en ligne le 01 septembre 2016, consulté le 15 avril 2022. URL : http://journals.openedition.org/ remi/6499; DOI : https://doi.org/10.4000/remi.6499 


\section{La fabrique du retour en Afrique. Politiques et pratiques de l'appartenance en Jamaïque (1920-1968)}

\section{Giulia Bonacci ${ }^{1}$}

L'abolition de l'esclavage en Jamaïque en 1834 laissa apparaître, pour reprendre les termes de Philip Curtin, deux Jamaïque, I'une caractérisée par un système culturel afro-jamaïcain qui prolongeait les pratiques populaires formées pendant l'esclavage, l'autre orientée vers un système culturel européen, compris comme le réveil de la civilisation britannique mise à mal durant l'esclavage - et qui caractérisent encore le pays (Patterson 1975 : 287). Dans ce contexte, les représentations de l'Afrique et les valeurs qui y étaient associées ont subi de nombreuses modifications, déployées entre la réappropriation et le rejet par une population en majorité noire ${ }^{2}$. La complexité historique des représentations de I'Afrique vue depuis la Jamaïque pourrait faire l'objet d'une étude approfondie mais nous nous limiterons ici à un mode particulier de ces représentations, qui fait de l'Afrique non seulement une terre d'origine mais aussi une terre de migration et de "retour ". Ainsi, des Jamaïcains, sujets coloniaux puis citoyens après l'indépendance de la Jamaïque en 1962, ont exprimé de manière régulière et tenace leur désir de migrer ou de " retourner " en Afrique, revendication faisant d'eux des " nationaux " en quête d'une autre nation que celle dans laquelle ils se trouvaient. La continuité historique de ce désir d'ailleurs n'occulte pas ses inconstances ni la variété des vocabulaires utilisés dans sa production. Ce " retour " en Afrique n'était pas une spécificité jamaïcaine dans la mesure où il s'inscrit dans un mouvement social plus général, Back to Africa movement, dont l'objectif était de "réparer " les déplacements de population dus à I'esclavage, d'échapper aux conditions socio-économiques ainsi qu'au statut colonial qui s'ensuivit. Ce retour, qui recouvre aussi la notion d'un " avant ", d'un ailleurs représentant un âge d'or, aurait le pouvoir de gommer les préjudices subis. En ce sens il s'agit d'une démarche idéologique, reconstructrice d'une origine, d'une

\footnotetext{
1 Chargée de recherche à I'Institut de Recherche pour le Développement (IRD), Unité de recherche Migrations et Société (URMIS) (UMR 205)/Centre français des études éthiopiennes (CFEE), P.O. Box 5554, Addis Abeba, Éthiopie ; giulia.bonacci@ird.fr

2 La proportion de descendants d'Africains dans la population jamaïcaine est estimée entre $76 \%$ et $91 \%$, ce qui place la Jamaïque parmi les îles les plus " noires " de la Caraïbe, au même titre que Haïti, la Guadeloupe, Sainte-Lucie, Saint-Vincent, Antigua et Grenade (Chivallon, 2004 : 66-67).
} 
trajectoire, des traces à suivre qui indiquent la route vers " chez soi ". La désignation d'un " chez soi ", d'une maison (home), d'une terre d'origine (fatherland, motherland, homeland) est liée aux sentiments d'appartenance, aux relations affectives et aux constructions identitaires caractéristiques des diasporas ${ }^{3}$. La question du retour, qui a nourri de manière significative les grandes idéologies produites dans les Amériques noires (éthiopianisme, nationalisme noir, panafricanisme), était l'objet de débats entre partisans de l'émigration vers l'Afrique et partisans de l'intégration aux Amériques. Si la prévalence, parfois qualifiée de "remarquable " (Shepperson, $1962: 356)$, des Caribéens dans les projets de retour et plus largement dans les discours et pratiques nationalistes et panafricains a déjà été mise en évidence (Bonacci, 2010 : 53-57), il s'agit dans cet article de revenir sur la place particulière de la Jamaïque dans les enjeux publics liés au retour.

Plus spécifiquement il s'agit de s'intéresser aux Jamaïcains qui ont formulé un désir de retour. On montrera d'une part que le " retour " a joué un rôle particulier, à la fois catalyseur social et politique, dans la naissance et le développement du mouvement rastafari jamaïcain, et d'autre part comment après l'échec de l'encadrement des demandes de retour par le gouvernement jamaïcain de petits groupes de rastafaris ont organisé leur départ vers l'Afrique à la fin des années 1960. En nous centrant sur la période qui va de 1920 à 1968, bornée d'une part par les archives accessibles sur le sujet et d'autre part par le premier " retour " volontaire d'un groupe de Jamaïcains en Éthiopie, c'est la fabrique du retour qui est ici à l'étude. C'est en exerçant un regard croisé entre les archives gouvernementales, la presse, I'histoire orale et la production écrite des demandeurs, notamment leurs lettres et pétitions, que nous souhaitons éclairer sous un nouveau jour les tensions entre les politiques et les pratiques de l'appartenance nationale ${ }^{4}$.

\section{Diversité sociale des demandeurs d'un retour en Afrique (1920-1940)}

\section{Les travailleurs engagés}

À la suite de l'émancipation des esclaves, effective en 1838 après les années " d'apprentissage " où les conditions des travailleurs n'avaient guère changé, les Britanniques se lancèrent dans une campagne d'engagement de milliers de Sierra Léonais qui vinrent travailler en Jamaïque. Ils furent par la suite nombreux à vouloir rentrer dans leur pays d'origine à la fin de leur contrat qui durait généralement cinq ans. Cependant, avec de faibles salaires et une baisse de leur niveau de vie en Jamaïque, quelques-uns seulement y parvinrent (Schuler, 1980 : 88-93). Entre 1843 et 1845, moins de 300 personnes firent le voyage par bateau. Parmi eux on notait la présence d'Africains libérés à Cuba, de vétérans des armées coloniales et d'enfants d'immigrants nés en Jamaïque. Puis avec une nouvelle période d'engagements contractuels, les autorités jamaïcaines se

\footnotetext{
3 En français, plusieurs ouvrages sont parus ces dernières années pour présenter les usages, les limites et les enjeux du concept de diaspora (Chivallon, 2004 ; Anteby-Yemini et al., 2005 ; Berthomière et Chivallon, 2006 ; Agudelo et al., 2009 ; Dufoix, 2011).

4 Cet article s'appuie en partie sur des résultats de recherche publiés en 2010 (Bonacci, 2010).
} 
désintéressèrent des questions liées au rapatriement des travailleurs. Entre 1849 et 1857,230 personnes au moins demandèrent à être rapatriées, mais seules quelques-unes firent le voyage. De nombreuses pétitions furent adressées de la Sierra Leone à la reine d'Angleterre pour demander le retour de parents exilés en Jamaïque. En 1861, soixante-quatorze personnes embarquèrent pour la Sierra Leone en payant elles-mêmes leur traversée alors que le gouvernement colonial n'envisageait aucune mesure dans l'aide aux retours. Ainsi des milliers d'immigrants restèrent en Jamaïque, nourrissant un imaginaire collectif de I'Afrique et du retour largement soutenu par l'internationalisation des migrations.

En effet, les migrations massives de Jamaïcains vers I'Amérique centrale et l'Amérique du Nord dès les années 1880 bouleversèrent le paysage social national et contribuèrent à internationaliser les pratiques et les expériences des Jamaïcains. Au début des années 1920, New York devint la première destination des migrants jamaïcains (James, 1998 : 49). Ce mouvement migratoire était particulièrement important car il permit aux migrants de se familiariser avec le langage international de l'unité raciale et de se forger une conscience panafricaine (James, 1998 : 71). Cette dernière prit de l'importance en Jamaïque ; la trajectoire de Marcus Garvey en fut exemplaire. Déjà engagé dans des activités syndicales en Jamaïque, il voyagea en Amérique centrale et séjourna à Londres avant de fonder I'Universal Negro Improvement Association (UNIA) à New York en juillet $1918^{5}$. Ses slogans, "Back to Africa ", "Africa for the Africans at home and abroad", avaient soulevé les espoirs des populations noires aux Amériques, qui subissait d'importants changements sociaux au début du XXe siècle, et la revendication du retour en Afrique se poursuivit après le déclin de I'UNIA à la fin des années 1920.

\section{Les vétérans du British West Indian Regiment (BWIR)}

Les vétérans caribéens ${ }^{6}$ qui avaient fait la Première Guerre mondiale sous commandement britannique avaient largement adhéré à I'UNIA. L'expérience de la ségrégation et de I'humiliation subie par ces soldats noirs au sein de l'armée britannique contribua à leur prise de conscience politique (James, 1998 : 56-60). Ainsi, toute une génération caribéenne se radicalisa, adoptant des idéologies plus raciales et nationalistes qu'auparavant. Ces positions apparaissent d'ailleurs clairement dans les demandes réitérées au gouvernement britannique afin de faciliter le retour en Afrique.

Le retour fut l'objet d'une première série de six lettres écrites en 1933 par Gilbert McKenzie, un natif de la Barbade, à l'attention du gouverneur de la Jamaïque et toutes signées, sauf la première, par plusieurs vétérans. McKenzie soulignait que ses ancêtres avaient été emmenés comme esclaves depuis Lagos jusqu'à la Jamaïque en 1806, un an avant l'abolition de la traite par les Anglais ${ }^{7}$.

5 Plus grande organisation noire de l'histoire, I'UNIA comptait 6 millions de membres en 1926 (Martin, $1986: 15-17)$.

6 Près de 15000 hommes furent recrutés, aux deux tiers des Jamaïcains, à la fondation en 1915 du British West Indian Regiment (BWIR), voir James (1998: 55-56).

7 Archives nationales de la Jamaïque, Spanish Town, [ANJ par la suite], 1B/5/77/394 [1933] Repatriation to Africa I (1933-1942), G. McKenzie à Sir Edward Denham, gouverneur de la Jamaïque, 20/05/1935 et G. McKenzie à B.H. Easter, secrétaire colonial, 28/05/1935. 
Il affirmait aussi qu'à ses côtés se trouvaient des descendants de Sierra Léonais qui avaient été recrutés comme travailleurs en 1826. Tous auraient servi dans le British West Indian Regiment (BWIR). À son retour en Jamaïque, McKenzie servit encore un temps sous les armes mais, après la dissolution du BWIR en 1927 et le krach financier de 1929, il se retrouva en difficulté et chercha du travail, sans succès. En dernier recours, il approcha le British West Indian Regiment Benevolent Fund, mais sans réussir à obtenir une aide sociale. L'indignation et la colère pointaient dans ses lettres. Dans un de ces premiers courriers il fit référence au retour en ces termes : " une déportation sans frais vers I'Afrique, la terre de mes pères, où je pourrai gagner ma vie pour améliorer ma condition frappée de pauvreté et regagner ce qui a été perdu ${ }^{8}$. Dans un courrier suivant il utilisa le terme rapatriement : "Nous demandons maintenant à être rapatriés en Afrique, chez nos ancêtres ${ }^{9}$. Deux semaines plus tard, il parla d'émigration : "Nous savons positivement, en pleine conscience d'esprit et avec des sentiments raciaux, que notre demande d'émigrer en Afrique est basée sur les droits raciaux d'une juste cause $"{ }^{10}$. Déporter, rapatrier, émigrer, dans tous les cas la responsabilisation du gouvernement colonial était demandée par McKenzie. Le gouverneur indiqua que " théoriquement, les arguments des pétitionnaires ne sont pas sans force et inspirent ma sympathie ${ }^{11}$. Mais la question du retour se heurtait à un problème majeur : "Si le gouvernement le reconnaissait [le droit au retour], nous devrions logiquement rapatrier tous les descendants d'esclaves africains qui réclament ce droit - un nombre qui pourrait facilement monter à des centaines de milliers ${ }^{12}$.

Et rapatrier des milliers de descendants d'Africains n'était pas dans les priorités du gouvernement britannique. Le 26 avril 1933, McKenzie reçut une réponse définitive : rapatrier la population était impraticable, il n'y avait pas de fonds disponibles et cette solution ne servait pas les intérêts des pétitionnaires. McKenzie réfuta ces arguments et continua à écrire, adressant même une pétition directement au roi à Londres ${ }^{13}$. En 1938, I'année des grandes révoltes sociales en Jamaïque, McKenzie continua de faire parvenir des pétitions au gouvernement colonial, ainsi que d'autres vétérans et d'autres personnes qui prirent le relais pour demander à rentrer en Afrique. Les lettres qui arrivèrent auprès du gouvernement colonial témoignaient visiblement d'une détresse sociale et les personnes concernées n'imaginaient d'autre solution que celle de partir vers le continent. À ces courriers, le gouvernement répondit systématiquement qu'il n'était pas dans ses objectifs de soutenir ces revendications, qu'elles n'étaient ni finançables ni désirables et les pétitionnaires ne se virent proposer aucune alternative.

\footnotetext{
8 ANJ, 1B/5/77/394 [1933], op. cit., G. McKenzie à Sir Alexander Ransford Slater, gouverneur de la Jamaïque, 12/03/1933.

9 ANJ, 1B/5/77/394 [1933], op. cit., G. McKenzie à Sir Alexander Ransford Slater, gouverneur de la Jamaïque, 20/03/1933.

$10 \mathrm{ANJ}, 1 \mathrm{~B} / 5 / 77 / 394$ [1933], op. cit., G. McKenzie à Sir Alexander Ransford Slater, gouverneur de la Jamaïque, 01/05/1933 et G. McKenzie au secrétaire colonial, 29/05/1933.

11 ANJ, 1B/5/77/394 [1933], op. cit., Minutes 14, 25/04/1933.

12 ANJ, 1B/5/77/394 [1933], op. cit., Minutes 14, 26/04/1933, mise en forme originale.

13 ANJ, 1B/5/77/394 [1933], op. cit., G. McKenzie et douze autres signataires au secrétaire d'État à Londres, 18/06/1935, 1ère lettre tapuscrite.
} 


\section{L'UNIA et la Repatriation Bill}

Avant d'être lancée à New York en 1918, I'Universal Negro Improvement Association (UNIA) avait eu un prédécesseur : I'UNIA and African Conservation Association and African Communities League, fondée en 1914 à Kingston en Jamaïque. Cette dernière se voulait une alternative à l'engagement politique local et proposait des programmes sociaux, culturels et musicaux (Hill, 1983 : Ixv). Après le déplacement du quartier général de I'UNIA à Harlem en 1918, jusqu'à onze branches locales se sont implantées en Jamaïque (Martin, 1986 : 16). Après la mort de Marcus Garvey à Londres en 1940, I'UNIA connut un certain déclin et sa visibilité internationale faiblit bien qu'en Jamaïque, le garveyisme resta très vivant. En 1937, une branche de I'UNIA, la Whitfield Division située à Trench Town, un quartier populaire de Kingston était réorganisée et, en septembre 1941, la secrétaire Florence Pitters écrivit au gouverneur Sir Arthur Richards pour l'informer que I'UNIA allait conduire un " travail missionnaire " dans le pays pour recueillir des signatures soutenant une proposition de loi sur le retour appelée Repatriation Bil/ ${ }^{4}$. En janvier 1942 parvenait au gouverneur de la Jamaïque la Repatriation Bill of Jamaica and the West Indies rédigée par Madame Pitters commanditée par le président général de I'UNIA, James R. Stewart ${ }^{15}$. Elle soulignait la similarité de destin entre les descendants d'Africains et les enfants d'Israël dont l'exode les libéra de l'esclavage égyptien : " Nous, les enfants opprimés descendants d'Africains, avons repris le cri - renvoyeznous en Afrique $"{ }^{16}$. L'archétype de l'Exode était présent dans les discours: les descendants d'Africains s'identifiaient aux Hébreux et le gouvernement britannique représenté par le Pharaon était implicitement menacé de futurs désastres. D'ailleurs en octobre 1942 Florence Pitters fit parvenir une copie de ce document directement au secrétaire d'État pour les colonies à Londres, dans la crainte que le gouverneur de la Jamaïque $\mathrm{n}^{\prime}$ informe pas ses supérieurs des revendications de I'UNIA. Elle affirma que I'UNIA avait 25000 membres enregistrés qui avaient signé cette pétition et qu'ils représentaient les 760000 Jamaïcains noirs ${ }^{17}$.

En 1944, alors que le transfert du pouvoir constitutionnel de la GrandeBretagne vers la Jamaïque était amorcé, la Whitfield Division relança ses demandes concernant le retour volontaire de la population vers le Libéria, cette fois avec un appel de fonds ciblé et une pétition unitaire, signée par différents groupes d'activistes. L'UNIA, " unie avec toutes les associations dans l'île travaillant pour le retour en Afrique ", en fait avec I'Afro-West Indian Welfare League, fondée en 1938 sous le nom de Negro Welfare League, présenta une pétition signée par 57860 " Afro-jamaïcains " qui demandait au gouvernement britannique un soutien financier leur permettant d'aller au Libéria où ils seraient les

14 ANJ, 1B/5/77/394 [1933], op. cit., Florence Pitters, UNIA, à Sir Arthur Richards, gouverneur de la Jamaïque, 24/09/1941.

15 ANJ, 1B/5/77/394 [1933], op. cit., Florence Pitters, UNIA, à Sir Arthur Richards, gouverneur de la Jamaïque, 14/01/1942 et ANJ, 1B/5/77/390 [1933] Repatriation to Africa II (19421946), Repatriation Bill of Jamaica and the West Indies.

16 ANJ, 1B/5/77/390 [1933], op. cit., Repatriation Bill of Jamaica and the West Indies, chapitre 8 et conclusion.

17, ANJ, 1B/5/77/390 [1933], op. cit., Florence Pitters à Sir Cosmo Parkinson, secrétaire d'État pour les colonies, 20/10/1942. 
bienvenus ${ }^{18}$. Les pétitionnaires reçurent une réponse du secrétaire colonial deux ans plus tard, sèche, courte et négative ${ }^{19}$. Cette mobilisation de la fin des années 1940 se solda par un échec collectif. Cependant certains Jamaïcains, ont réussi à financer individuellement leur voyage et réussirent à partir pour le Libéria et plus largement vers I'Afrique de I'Ouest, sans que I'histoire de leurs installations ne soient connues (Campbell, $1994: 85)^{20}$.

Les lettres et pétitions qui affirmaient regrouper des dizaines de milliers de signatures gonflèrent très probablement leurs chiffres dans l'espoir d'attirer l'attention du gouvernement. Pourtant, même exagérés, ils démontraient la vivacité de l'imaginaire lié au retour vers "l'Afrique ", continent pourtant méconnu, hormis le Liberia qui occupait une place centrale dans l'imaginaire des garveyites, alors que l'usage du nom Éthiopie ne servait le plus souvent qu'à désigner leur appartenance raciale. Ce ne fut qu'avec les rastafaris que la référence à l'Éthiopie prit de l'importance. Les propos d'un rastafari jamaïcain arrivé en Éthiopie en 1973 illustrent ce recentrement :

"-C'est là [auprès des rastafaris] que j'ai eu ma petite sagesse à travers ces petits raisonnements, alors j'ai été enraciné dans l'Afrique, parce que les hommes qui étaient assis là parlaient deux, trois heures, beaucoup de gens étaient là pour parler, tu sais.

- Ceux qui étaient assis étaient des rastaman?

— Oui ! (...) C'était des garveyites, des bedwardites, et des binghi, des Fédérations (...). Tout le monde parlait d'Afrique et là on a décidé de venir en Afrique.

- Certains parlaient du Libéria?

— Ils parlaient du Libéria, de la Sierra Leone, du Ghana, mais l'Éthiopie ils en parlaient mieux que du Ghana et de tous les autres pays. L'Éthiopie je te dis $»^{21}$.

Les rastafaris et les militants du retour en Afrique dans les années 1930 et 1940 tissèrent de nombreux liens malgré la diversité des trajectoires individuelles. Dans les assemblées de rastafaris tous se retrouvaient pour parler d'Afrique malgré les différentes affiliations qui existaient (à Marcus Garvey le nationaliste, à Alexander Bedward, figure prophétique active en Jamaïque au début du XXe siècle, aux groupes rastafaris nyahbinghi et Ethiopian World Federation). De tous les pays d'Afrique qu'ils pouvaient mentionner l'Éthiopie occupait une place particulière et devenait une référence majeure dans le mouvement rastafari en pleine construction.

\section{Les rastafaris et le retour en Afrique}

\section{Leonard P. Howell}

Pour les rastafaris, le rapatriement en Afrique avait une double fonction, à la fois nationaliste et religieuse : il était à la fois le moyen de territorialiser

18 ANJ, 1B/5/77/367 [1933] Repatriation to Africa III (1946-1951), Florence Pitters au gouverneur et au conseil exécutif, 04/08/1948 et pétition unitaire avec I'Afro-West Indian Welfare League, 05/07/1948.

19 ANJ, 1B/5/77/367 [1933], op. cit., secrétaire colonial à Florence Pitters, 20/05/1950.

20 En 1949 Le PNP (People's National Party) devient un parti de masse qui défendait le " socialisme démocratique ".

21 Entretien avec E. Reid, Shashemene, Éthiopie, 02/04/2003. 
la nation noire et celui de concrétiser leur rédemption. Outre la démarche qui consistait à exiger un droit dû à leur statut de descendants d'esclaves africains, ils renouèrent avec les prophéties bibliques qui annonçaient le retour à Sion et firent de la "réalisation de la prophétie " un objectif central, parfois d'ailleurs plus rhétorique que pratique. Le passage biblique peut-être le plus souvent cité pour justifier le rapatriement comme dessein providentiel était tiré du livre d'Isaïe (XLIII, 5-7). Le langage de I'Exode, I'identification aux enfants d'Israël et leur retour à Sion furent recontextualisés pour répondre aux conditions économiques et sociales et aux expériences de la discrimination des rastafaris jamaïcains. Les rastafaris proposèrent en fait une allégeance nationale non à I'empire britannique mais à l'empire éthiopien. Alors que Leonard Howell, souvent désigné comme le " premier " rastafari (Lee, 1999) était en prison pour sédition en 1934, on pouvait lire dans la presse que les rastafaris se préparaient à rentrer en Éthiopie en marchant sur les eaux (Van Dijk, 1993 : 92). Plus sérieusement, il fallut attendre les années 1940 pour que leurs revendications remontent jusqu'au gouvernement de manière officielle.

\section{Plaidoyer pour un retour}

Entre 1943 et 1949, trente-cinq lettres au moins, pour la plupart manuscrites, plaidant la cause du retour ont été adressées au secrétariat colonial. Le vocabulaire utilisé, à la fois religieux et nationaliste faisait référence explicitement à " notre Dieu glorieux, roi Rastafari "22, au " Dieu et roi des Noirs [qui] est ici et qui est le roi de la gloire ${ }^{23}$, à " notre gouvernement noir, roi et empereur d'Éthiopie ${ }^{24}$, ou encore à " Hailé Sellassié ler, pouvoir de la Trinité " ${ }^{25}$.

C. Jones avait été chauffeur pour le JLP (Jamaica Labour Party) dans les années 1940 mais sans travail depuis trois ans, il suppliait le gouvernement de lui trouver un emploi dans I'attente de son retour en Afrique ${ }^{26}$. II avait fait parvenir nombre de courriers manuscrits tous emprunts de références bibliques et qui commençaient par "Nous, les enfants d'Éthiopie... " ${ }^{27}$. II reprenait des extraits de psaumes comme celui bien connu (psaume 137) qui débute par "Au bord des fleuves de Babylone nous étions assis et nous pleurions, nous souvenant de Sion... ${ }^{28}$. La plainte des Israélites exilés était réappropriée pour faire du départ en Éthiopie un retour appelé par Dieu, ici le " roi Rastafari ". La dimension nationaliste transparaissait dans ses écrits et mentionnait Hailé Sellassié ${ }^{\text {er. }}$. D'autres demandeurs comme B. L. Wilson qui manifestait son

22 ANJ, 1B/5/77/390 [1933], op. cit., C. Jones au secrétaire colonial, 28/04/1948.

23 ANJ, 1B/5/77/390 [1933], op. cit., Dawson African à Sir John Huggins, gouverneur de la Jamaïque, 21/05/1945.

$24 \mathrm{ANJ}, 1 \mathrm{~B} / 5 / 77 / 390$ [1933], op. cit., R. E. Bennett et M. L. Henderson à Sir John Huggins, gouverneur de la Jamaïque, 15/12/1943.

25 ANJ, 1B/5/77/390 [1933], op. cit., B. L. Wilson au secrétaire colonial, 01/09/1944, 15/09/1944, 20/10/1944 et 05/01/1945.

26 ANJ, 1B/5/77/390 [1933], op. cit., C. Jones au secrétaire colonial, 05/06/1943.

27 ANJ, 1B/5/77/390 [1933], op. cit., C. Jones au secrétaire colonial, 28/04/1943.

28 Ce psaume était depuis longtemps part du répertoire musical sacré des rastafaris, le nyahbinghi. Mis en musique en 1969 par le groupe les Melodians qui en fit un classique du reggae, Rivers of Babylon, il était repris en 1978 par le groupe jamaïcain de disco

Boney $\mathrm{M}$, qui le transforma à son tour en un succès international. 
attachement à " un roi, un pays et un drapeau " éthiopiens ${ }^{29}$, ou Egbert Smith, qui dans ses lettres, mentionnait les " droits nationaux et le respect " que les noirs voulaient obtenir ${ }^{30}$, écrivaient depuis Dunghil/1, un quartier de Kingston qui était devenu un des premiers bastions du mouvement rastafari. Des références à I'histoire de l'esclavage étaient souvent faites par les pétitionnaires pour démontrer qu'ils étaient dans leur droit et prouver que leurs revendications étaient justes : "I'histoire prouve que nos ancêtres ont été emmenés comme esclaves depuis notre terre mère l'Éthiopie ${ }^{32}$. Cette certitude d'avoir eu des ancêtres arrachés à l'Éthiopie était une constante chez les rastafaris et servait à " concrétiser " la filiation imaginée entre l'Éthiopie et les peuples noirs et, par conséquent, à légitimer le retour vers l'Éthiopie tout particulièrement.

Toutes ces demandes liées au retour en Afrique témoignaient de I'attachement des rastafaris à l'Éthiopie à travers la divinité de l'empereur et les attributs de la nation éthiopienne. Ce faisant, ils défiaient le gouvernement colonial jamaïcain. Le désir de retour devenait l'objet de revendications constantes dans une société en pleine transformation politique. Au milieu des années 1950, alors que des terres à Shashemene, dans le sud de l'Éthiopie, étaient mises à disposition par Hailé Sellassié ler, le mouvement rastafari pris de l'ampleur. En devenant concret, l'espoir d'un territoire rendit visible la lutte des rastafaris mais provoqua aussi de vives tensions sociales.

\section{Les acteurs du retour}

\section{L'Ethiopian World Federation (EWF)}

Fondée en 1937 à New York par l'Éthiopien Melaku Beyen et sur ordre de I'empereur Haile Sealssie, alors en exil en Angleterre, I'Ethiopian World Federation (EWF, Fédération mondiale éthiopienne) avait comme mission de centraliser le soutien moral et financier offert à l'Éthiopie par les noirs des Amériques pendant la guerre italo-éthiopienne (Bonacci, 2010 : 127-131; Bonacci, 2013). En 1955 la soprano américaine Mayme Richardson se rendit en Jamaïque pour annoncer que des terres étaient disponibles en Éthiopie pour les membres de I'EWF. Cette annonce eut comme effet la multiplication des branches locales de l'EWF en Jamaïque dont une douzaine s'ouvrit à l'initiative de groupes nouvellement formés ou de congrégations existantes. Alors que I'EWF aux États-Unis était à ses débuts une organisation à caractère œcuménique, en Jamaïque de vives tensions apparurent à propos de l'appartenance religieuse. Les rastafaris n'arrivaient pas à trouver leur place au sein de l'EWF alors qu'ils n'étaient pas prêts d'une part à mettre leur foi en sourdine et d'autre part parce que les officiers de la Fédération qui géraient les relations administratives de I'EWF entre la Jamaïque et New York, n'étaient pas des rastafaris et les tenaient à l'écart. L'EWF était une organisation fortement structurée et la gestion administrative des branches locales, peu rigoureuse, était un obstacle

29 ANJ, 1B/5/77/390 [1933], op. cit., B. L. Wilson au secrétaire colonial, 05/01/1945.

30 ANJ, 1B/5/77/390 [1933], op. cit., Egbert Smith au secrétaire colonial, 14/05/1943.

31 Littéralement montagne d'excréments, parfois appelé aussi " Dungle ", une contraction de dung et jungle, en fait une grande décharge d'ordures où vivaient les indigents. 32 ANJ, 1B/5/77/390 [1933], op. cit., R. E. Bennett et M. L. Henderson à Sir John Huggins, gouverneur de la Jamaïque, 12/10/1943. 
à la réalisation d'un programme collectif. Aurait-il pu en être autrement au sein d'une population, mise à l'écart par les branches les plus influentes de I'EWF, en partie illettrée, sans compétences administratives et dont les attentes mystiques ne jouaient pas en faveur d'actions concrètes ? Cependant, l'annonce d'un don de terres en Éthiopie devint un puissant argument en faveur du retour et c'est après 1955 que le mouvement rastafari investit l'EWF et s'adressa directement au gouvernement jamaïcain.

\section{Prince Emmanuel}

En mars 1958 un rastafari du nom de Prince Emmanuel Charles Edwards appela à la tenue d'un grand congrès appelé " convention " ou " groundation ". II s'était déjà fait remarquer auprès des autorités coloniales une dizaine d'années auparavant en écrivant au secrétariat colonial pour demander assistance en vue de rentrer en Afrique ${ }^{33}$. II décrivait dans ses lettres les conditions de surpopulation, de malnutrition et de pauvreté des "sufferers " et parlait d'un " gigantesque mouvement " vers l'Afrique ou l'Éthiopie. Le grand congrès se tint dans le centre de Kingston. Durant vingt et un jours, des milliers de personnes se rassemblèrent au son des tambours nyabinghi et allumèrent de grands feux $^{34}$. La presse jamaïcaine assista quelque peu horrifiée à ce rassemblement de rastafaris venus de toute l'île. La question du rapatriement était à l'ordre du jour et de nombreux rastafaris avaient vendu leurs biens avec la certitude qu'ils pourraient embarquer pour l'Afrique à la fin du congrès (Chevannes, 1994 : 173). Ce rassemblement réunit des rastafaris de toute l'île et de différents groupes, donnant aussi une certaine notoriété à Prince Emmanuel. II marqua " le moment décisif dans la détérioration des relations entre d'un côté le gouvernement et la société, et de l'autre le mouvement Ras Tafari " (Smith et al., 1960 : 15) et après le congrès, la pression policière s'accentua. Prince Emmanuel vit son camp brûlé par la police et fut arrêté, emprisonné, jugé puis finalement libéré (Van Dijk, 1993 : $118 ; 146-149)$.

\section{Claudius Henry}

En 1959, une altercation entre rastafaris et la police au Coronation Market, le grand marché au cœur de Kingston se solda par près d'une centaine de personnes emprisonnées et de nombreuses habitations détruites dans les ghettos de Ackee Walk et Back-O-Wall (Van Dijk, 1993 : 118-123). À la faveur de ces violences, le révérend Claudius Henry se fit connaître. Jamaïcain de retour d'un séjour de treize ans aux États-Unis, il essaya sans succès de s'associer à l'EWF et fonda par la suite son église, The Seventh Emmanuel Brethren, renommée African Reform Church. II se fit appeler le "réparateur des brèches " (Isaïe LVIII), et fit circuler des milliers de petites cartes bleues qui portaient quelques lignes: "Aucun passeport ne sera nécessaire pour ceux qui rentrent à la maison en Afrique. Prenez ce certificat avec vous le $1^{\text {er }}$ août pour "identification" ${ }^{35}$. Des milliers de personnes se retrouvèrent à cette date sur le port de Kingston après

33 ANJ, 1B/5/77/367 [1933], op. cit., Prince Emmanuel Charles Edwards, à Sir John Huggins, gouverneur de la Jamaïque et au secrétaire colonial, cinq lettres datées 07/07/1948 et 24/01/1950, deux le 13/09/1950 et le 21/09/1950.

34 Jamaica Times, National Library of Jamaica, 08/03/1958, p. 1.

35 Public Record Office, Londres [PRO par la suite], CO 1031/2768, où se trouve un original de cette carte bleue. 
avoir vendu leurs biens et abandonné leurs logements dans l'espoir d'un " rapatriement miraculeux ". Le Daily Gleaner titra le lendemain, "Pas de passeports, pas de réservation ; mais ils "rentrent en Afrique" ", soulignant ainsi I'absence de préparation réelle et le caractère frauduleux de cette initiative ${ }^{36}$. Passé la date limite, les migrants potentiels s'en retournèrent, amèrement déçus, quelques centaines refusant même de quitter le port parce qu'ils n'avaient pas les moyens de se déplacer ou par honte de devoir assumer leur échec. Henry fut envoyé en prison et dut s'acquitter d'une amende. À la fin de l'année des armes étaient découvertes chez son fils et chez certains de ses disciples (Chevannes, 1976). Accusés de trahison et de préparation d'un coup d'état soutenu par la révolution cubaine victorieuse, Henry et une quinzaine de personnes furent alors jugés lors d'un procès largement médiatisé et qui fut l'occasion aussi de débattre du " retour en Afrique ".

\section{Mortimer Planno}

Certains des leaders du mouvement rastafari commencèrent à faire arriver leurs revendications de retour à la presse panafricaine ce qui leur permettait de s'adresser à un public plus large qui ne connaissait pas les rastafaris. C'est à cette période que Mortimer Planno, aussi connu sous le nom de Ras Kumi, né en 1929 à Cuba mais arrivé en Jamaïque avec ses parents en 1932, se fit remarquer aux côtés de Prince Emmanuel, comme un des membres qui allaient compter dans le milieu des rastafaris. Plusieurs de ses courriers ont été publiés par African Opinion où il concentra son attaque contre la violence du gouvernement jamaïcain. II l'accusa de ne pas reconnaître la Déclaration universelle des droits de I'homme du 10 décembre 1948, en s'appuyant sur les articles 3, 5, 6, et 7 pour démontrer que les persécutions, dont les rastafaris étaient l'objet, étaient contraires au droit international. Parlant au nom d'une fédération de cultes appelée United African Movement il déclara :

"Nous avons décidé de marquer ce jour [10 décembre] pour réclamer notre nationalité - éthiopienne ou africaine - en accord avec la charte des Nations unies (...) nous avons tous les droits pour demander notre nationalité - éthiopienne où notre roi est notre Tout-puissant $\aleph^{37}$.

Selon I'article 15 qui spécifie : " Tout individu a droit à une nationalité. Nul ne peut être arbitrairement privé de sa nationalité, ni du droit de changer de nationalité ", aucun obstacle n'interdisait dès lors d'acquérir la nationalité éthiopienne. Planno dans un autre courrier déclara qu'il n'y avait pas d'autre solution que "le rapatriement ou la rébellion armée " et il signifia que cet ultimatum concernait " 45000 nationalistes africains et des milliers de frères et sœurs ${ }^{38}$. La carrière publique de Mortimer Planno était lancée et il joua par la suite un rôle important dans le changement de regard que la société jamaïcaine portait sur les rastafaris.

36 Daily Gleaner [DG par la suite], National Library, Kingston, Jamaïque [NLK par la suite], 06/10/59.

37 African Opinion [AO par la suite], UCLA Research Library [UCLA par la suite], 1959, 4, 11 et 12 , pp. 6-7.

38 AO, UCLA, 1959, 5, 3 et 4, p. 9. 


\section{Les soubresauts politiques de l'appartenance nationale}

\section{Le rapport sur le mouvement rastafari de 1960}

Trois universitaires, M. G. Smith, R. Augier et R. Nettleford conduisirent une rapide enquête sur I'histoire du mouvement, ses spécificités doctrinales et ses organisations, dont la " composition complexe et l'hétérogénéité des éléments" les laissèrent "parfois pantois" (Smith et al., $1960: 29)$. Connue sous le nom de " rapport de 1960 ", cette enquête a souvent été considérée comme une histoire définitive du mouvement rastafari alors que des recherches conduites par Robert A. Hill ${ }^{39}$ démontrent aujourd'hui que c'était une initiative du ministère de I'Intérieur, dont l'objectif était de " pacifier " le mouvement rastafari et de prévenir sa radicalisation, représentée notamment par Claudius Henry. L'enquête conclut au fait que les rastafaris n'étaient ni des criminels ni des malades mentaux, contrairement à ce qui circulait le plus souvent dans la presse, mais soulignait néanmoins la menace représentée par la diffusion d'idées marxistes sous couvert de mouvement rastafari. Les universitaires firent état des conditions socio-économiques déplorables dans lesquelles vivaient la plupart des rastafaris et rendirent publiques les revendications de retour en Afrique. Ils expliquèrent les raisons de leur position en ces termes:

«1. Chaque citoyen a le droit d'émigrer s'il le désire, et de changer sa nationalité s'il le désire.

2. Alors que beaucoup de frères Ras Tafari resteraient en Jamaïque s'ils trouvaient du travail et de bonnes conditions sociales, un grand nombre d'entre eux ont de forts liens religieux avec l'Afrique qui ne peuvent être détruits.

3. La Jamaïque est surpeuplée et ne peut offrir du travail à tous ses citoyens. Tous les efforts devraient être faits pour faciliter l'émigration.

4. La Jamaïque facilite actuellement I'installation d'émigrants en Angleterre ; d'un point de vue racial l'émigration vers I'Afrique semble plus appropriée.

5. Une émigration substantielle vers l'Afrique ne sera pas possible à moins que le gouvernement jamaïcain ne prenne certaines initiatives " (Smith et al., 1960 : 34).

Pour la première fois, l'élite intellectuelle se fit l'écho des revendications des rastafaris et adressa directement au gouvernement des recommandations dont la première avait trait au retour en Afrique : " Le gouvernement jamaïcain devrait envoyer une mission dans des pays africains afin de prendre des dispositions pour l'immigration de Jamaïcains. Des représentants des frères rastafaris doivent être inclus dans cette mission " (Smith et al., 1960 : 38). Cette recommandation, négociée au préalable avec le gouvernement jamaïcain, et présentant la mobilité vers I'Afrique comme une " migration " et non comme un " retour ", devait servir à " coopter " des rastafaris et, dans les termes du Premier ministre N. Manley, " les faire revenir sur terre " (Hill, 2013). L'impact du " rapport de 1960 ", publié plusieurs semaines de suite dans la presse quotidienne, fut considérable et si aucune des politiques sociales faisant l'objet des huit autres recommandations

\footnotetext{
39 Robert A. Hill avait accusé ce rapport de nourrir une « myopie historique " qui aurait limité la recherche sur I'histoire et la formation du mouvement (Hill, 2001 : 13-14). Le résultat de ses travaux, présentés récemment au public, va beaucoup plus loin et documente la supercherie que représente ce rapport, qu'il qualifie d'opération de " contre-insurrection " pilotée par les services de renseignement du gouvernement jamaïcain. Voir Hill (2013).
} 
ne fut mise en place au profit des rastafaris, une mission fut effectivement mise sur pied pour étudier les possibilités d'accueil des Jamaïcains en Afrique.

\section{La première mission vers l'Afrique}

Dès la fin de l'année 1960, le Premier ministre jamaïcain Norman Manley (PNP, People's National Party), amorça des discussions en vue d'une mission non-officielle et semi-gouvernementale vers I'Afrique afin d'y étudier les possibilités concrètes d'émigration et d'accueil des Jamaïcains, en Éthiopie, au Nigeria, au Ghana, au Libéria et en Sierra Leone. Alors que pour la première fois une occasion se présentait d'avoir une position commune et de choisir une délégation représentative du mouvement, les rastafaris se divisèrent sur des questions de leadership, de représentativité et de différences doctrinales. La sélection des autres délégués fut elle aussi houleuse, et, finalement, neuf personnes furent du voyage : Mortimer Planno, Douglas Mack et Filmore Alvaranga pour le mouvement rastafari, Westmore Blackwood de I'UNIA, Dr. B. Douglas de I'AfroCaribbean League, Cecil Gordon de I'Ethiopian World Federation et Munroe Scarlett de I'Afro-West Indian Welfare League. Ils étaient accompagnés d'un journaliste, Victor Reid et du Dr. Leslie, un médecin (Majority Report, 1961 : 1). La présence de ces différentes organisations dans la mission montre clairement que I'activisme lié au retour en Afrique n'était pas limité aux rastafaris, mais était partagé par des militants connus. Les tensions ayant entouré l'élection de ces représentants ne laissèrent pas présager d'une entente parfaite entre eux, et le clivage entre rastafaris et non-rastafaris se retrouva jusque dans les rapports produits suite à la mission : un rapport " minoritaire ", signé par les rastafaris et un rapport " majoritaire ", signé par les autres ${ }^{40}$.

La mission arriva en Éthiopie en avril 1961 et fut reçue avec les honneurs à Addis-Abeba. En une semaine, elle effectua de nombreuses visites auprès d'officiels de l'Église orthodoxe éthiopienne et du gouvernement dans la capitale et en province, jusqu'à Shashemene. Après avoir rencontré Abuna Basileos, le patriarche de l'Église, les membres de la mission furent reçus par l'empereur: "Sa Majesté Impériale nous a dit qu'il connaissait les Noirs de l'ouest et en particulier la Jamaïque, frères de sang des Éthiopiens et il savait que des esclaves avaient été emmenés depuis l'Éthiopie en Jamaïque. II dit que nous devions envoyer les bonnes personnes. L'empereur dit que l'Éthiopie était assez grande pour contenir tous les peuples descendants des Africains qui vivaient hors d'Afrique " (Minority Report, $1961:$ 16-17).

Ces mots furent souvent repris, réutilisés, réinterprétés par les rastafaris dans de multiples situations. Que l'empereur reconnaisse un lien consanguin était un signe très fort, une " confirmation " pour tous les Jamaïcains qui se considéraient Éthiopiens. L'expression "les bonnes personnes " a aussi été largement répétée et discutée, avec de multiples argumentations sur le sens recouvert : est-ce que les rastafaris étaient "les bonnes personnes " ? Encore aujourd'hui les rastafaris font référence à ces propos.

40 Ces deux rapports sont différents dans le format adopté, le rapport majoritaire présentant des paragraphes de synthèse par pays, et le rapport minoritaire suivant un découpage au quotidien. Dans le contenu, le rapport minoritaire retrace avec plus de détails les entrevues avec l'empereur Hailé Sellassié ler, mais il n'y a pas de contradictions importantes entre les deux documents. 
La mission continua son voyage vers le Nigeria, où ils rencontrèrent entre autres Nnamdi Azikiwe, alors gouverneur général. Il leur parla de la dette qu'il avait envers les Caribéens : "II remarqua qu'il avait lui-même eu des professeurs Caribéens (West Indians) et affirma que la philosophie de Marcus Garvey était en grande partie responsable de son travail vers l'indépendance du Nigeria " (Majority Report, 1961 : 6). Ministres et chefs coutumiers leur assurèrent que des installations pourraient se faire, que le retour en Afrique était une nécessité et que les Caribéens étaient des Africains. La mission repartit ensuite pour le Ghana. Elle fut reçue par le président Kwame Nkrumah qui affirma : "Comment le dire ? Notre rencontre est historique. Elle a une signification historique non seulement parce que nous sommes des relations de sang mais aussi parce que de nombreux essais ont été faits auparavant mais sans succès. Marcus Garvey essaya mais il en a été empêché " (Majority Report, 1961 : 9). Puis Nkrumah assura que des terres pourraient être mises à disposition. Le même accueil chaleureux eut lieu au Libéria, où la mission fut reçue par le président Tubman, qui se montra clair sur le fait que le Libéria avait des lois facilitant l'immigration. Après treize jours au Libéria la mission se rendit en Sierra Leone. Le Premier ministre, Dr. Sir Milton Margai confirma que sur le principe la Sierra Leone était ouverte à des projets de retour, mais il indiqua néanmoins que l'indépendance du pays ayant été obtenue moins d'un mois auparavant (le 27 avril 1961) le gouvernement avait à l'heure actuelle des préoccupations plus urgentes. Dix jours plus tard, la mission rentra en Jamaïque. 5000 personnes environ étaient à l'aéroport pour les accueillir avec bannières, drapeaux, chants et tambours.

\section{La mission technique de 1962}

Suite à la mission de 1961, une autre mission fut envoyée par le gouvernement entre janvier et avril 1962 au Nigeria, au Ghana et en Éthiopie. Composée de seulement quatre personnes, sans rastafaris, elle devait étudier "les problèmes techniques posés par le projet de déplacement de personnes depuis la Jamaïque vers les nations de l'Afrique " (Nettleford, 2001 : 70). Rex Nettleford, qui avait cosigné le rapport de 1960 sur le mouvement rastafari faisait partie de cette mission, comme Aston Foreman, qui allait devenir en 1970 le premier ambassadeur de la Jamaïque en Éthiopie. La mission technique spécifia que la plupart des nations ouest africaines préféraient accueillir des Jamaïcains qualifiés et professionnels alors que le gouvernement éthiopien semblait prêt à mettre à disposition des terres pour des agriculteurs jamaïcains. Les frais afférents à l'installation de familles jamaïcaines à Shashemene, en Éthiopie, étaient présentés au gouvernement (Technical Mission to Africa, 1962 : 89-90; 101). Le rapport de cette mission passa inaperçu dans la presse et le changement de gouvernement contribua à enterrer ses conclusions (Nettleford, 2001 : 71).

En Avril 1962, le JLP (Jamaica Labour Party), principal parti d'opposition, conduit par Alexander Bustamante, remporta les élections et le gouvernement ouvrit les festivités d'indépendance le 6 août 1962. Dans ce contexte il y eut peu de place pour des forces alter-nationalistes comme les rastafaris ${ }^{41}$. Leur souhait

41 Van Dijk (1993: 164) qualifia d' « anti-nationalistes " les forces représentées par les rastafaris. II nous semble pourtant qu' " alter-nationaliste " serait plus adéquat, car ce $\mathrm{n}^{\prime}$ est pas toute allégeance nationale qui était refusée par les rastafaris, mais spécifiquement I'allégeance - I'appartenance - à la nation jamaïcaine. 
d'obtenir la nationalité éthiopienne allait de pair avec le rejet de la nationalité jamaïcaine et fut proportionnel au sentiment d'aliénation qu'ils ressentaient dans la société insulaire (Smith et al., 1960 : 21). Ils s'exprimèrent tout particulièrement par rapport à la devise de la nouvelle nation, "Out of many, one people ", qui se voulait à l'image du multiculturalisme ou du non racialisme supposé de la Jamaïque. Cette idéalisation de la nation jamaïcaine, portée par la classe moyenne, fut contestée par les rastafaris et accusée d'ignorer les contributions du large substrat de la population noire dans l'île dont ils se faisaient les porte-paroles (Nettleford, 2001 : 19-37). Alors que les rastafaris étaient perçus comme une menace à l'identité nationale qui prenait forme sous le gouvernement post-colonial, I'année 1963, avec le " massacre de Coral Gardens " (Van Dijk, 1993 : 164-169) a été marquée par une violente répression contre le mouvement rastafari.

\section{La deuxième mission vers I'Afrique}

Trois rastafaris décidèrent de lancer une deuxième mission vers l'Afrique, mais sans bénéficier du soutien du gouvernement jamaïcain. Ils menèrent campagne pour trouver des fonds et réussirent à partir pour New York en décembre 1963. Filmore Alvaranga et Douglas Mack avaient participé à la première mission, et Samuel Clayton était un talentueux musicien nyabinghi. Munis d'une pétition qui listait les métiers et compétences des rastafaris désireux d'émigrer, ils furent accueillis à New York par Gladstone Robinson, un Américain (Cherokee et Barbadien d'origine) membre de l'EWF et militant du retour en Afrique qui partit en 1964 pour l'Éthiopie. Ils formèrent ensemble I'African Repatriation Committee $(A R C)$ et rassemblèrent les fonds nécessaires pour continuer le voyage. Arrivés au Nigeria, ils furent logés par le gouvernement qui au bout de deux semaines était prêt à les renvoyer en Jamaïque sans leur avoir accordé d'audience. Devant le refus catégorique des rastafaris, un billet d'avion pour le Kenya leur fut offert. Ils y restèrent quatre mois, logés sur ordre du président Jomo Kenyatta qui finança ensuite leur billet d'avion pour l'Éthiopie où ils arrivèrent en novembre 1964 et furent accueillis par le clergé orthodoxe. Ce n'est qu'en avril 1965 qu'ils furent officiellement reçus par l'empereur, qui leur confirma que l'Éthiopie pourrait accueillir les rastafaris qui voudraient s'y installer (Mack, 1999 : 141-142). À leur retour à Kingston ils fondèrent la Rastafarian Brethren Repatriation Association en mai 1965 qui venait s'ajouter à la longue liste d'organisations plus ou moins efficaces qui organisaient le mouvement rastafari.

\section{La visite de Hailé Sellassié ler en Jamaïque}

L'annonce d'une visite officielle d'Hailé Sellassié ler en Jamaïque prévue pour avril 1966 souleva l'enthousiasme des rastafaris et l'espoir d'un rapatriement immédiat. La tournée caribéenne de l'empereur le mena en Haïti, puis en Jamaïque et finalement à Trinidad après un passage à la Barbade. Son arrivée à Kingston le 21 avril déclencha une grande mobilisation populaire. Dès la veille, près de 10000 personnes s'étaient rassemblées sur le tarmac de l'aéroport sans que la force publique n'intervienne en cette période préélectorale. À peine l'empereur arrivé, " la foule bondit depuis les toits des bâtiments de l'aéroport, sauta les barrières et se répandit vers I'avion, débordant les VIP officiels, la 
police, la garde d'honneur et l'orchestre ${ }^{42}$. Profondément bouleversé par cet accueil l'empereur eut bien du mal à sortir de l'avion et il fallut toute l'autorité de Mortimer Planno pour que la foule le laisse passer. Le moment était historique pour les Jamaïcains réunis en masse et pour les rastafaris qui furent invités à participer aux réceptions organisées en I'honneur d'Hailé Sellassié ler et se retrouvèrent, pour la première fois, à côté des invités de marque et des notables de l'île à la King's House de Kingston. L'empereur accorda une audience aux rastafaris qui avaient participé aux missions de 1961 et 1963, ainsi qu'aux représentants de différents groupes rastafaris et d'organisations militantes.

Le gouvernement jamaïcain espéra en vain qu'à l'occasion de cette visite Hailé Sellassié ler désavoue la foi placée en lui par les rastafaris (Van Dijk, 1993 : 175). Au stade national, prenant la parole après Donald Sangster alors Premier ministre, l'empereur s'exprima en ces termes :

" Je connais l'amour que les Jamaïcains ont pour le peuple d'Éthiopie et l'accueil que vous m'avez donné ce jour en est la preuve. Je sais que les Jamaïcains étaient très proches de l'Éthiopie quand elle était occupée durant le régime fasciste. À ce moment-

là, les Jamaïcains et d'autres qui avaient du sang africain ont soutenu le mouvement éthiopien de libération. (...) Je sais que vous partagez les sentiments des Africains. Les Jamaïcains et les Éthiopiens sont des frères de sang et ont eu une relation qui remonte à très longtemps $n^{43}$.

En faisant référence au grand moment panafricain de 1935-1941 et en mentionnant non seulement le sang africain des Jamaïcains mais aussi le sang commun des Éthiopiens et des Jamaïcains, l'empereur était certain de s'attacher une admiration sans borne de la part de ses supporters jamaïcains. Ces paroles furent reçues comme une légitimation et une preuve de la véracité des revendications des rastafaris qui demandaient depuis longtemps la nationalité éthiopienne. Après cette visite, les Britanniques considérèrent que l'importance de la mobilisation populaire était un signe de la faiblesse de la sécurité nationale. Ils soulignèrent également la peur créée au sein de la classe moyenne jamaïcaine qui voyait dans ces manifestations " le présage qu'ils avaient toujours craint le plus - l'élan insoumis des masses noires d'en bas " ${ }^{44}$. Mais pour ces " masses noires ", rencontrer un roi africain, descendant d'une lignée révérée fut une expérience marquante ; pour les rastafaris ce moment fut proche d'une parousie, l'apparition du Dieu vivant, et de leurs yeux ils pouvaient voir la personne qu'ils révéraient.

42 PRO, DO 200/170, Rapport confidentiel de J. D. Murray, haut commissionnaire britannique en Jamaïque à l'attention du secrétaire d'État pour le Commonwealth, 14/06/66, p. 3.

43 Reggae \& African Beat, UCLA, 1986, 5 (5-6), Discours de Hailé Sellassié ler en Jamaïque, 21 avril 1966.

44 PRO, DO 200/170, Rapport confidentiel de J. D. Murray, haut commissionnaire britannique en Jamaïque à I'attention du secrétaire d'État pour le Commonwealth, 14/06/1966, p. 4 . 
Les Britanniques déclarèrent après le départ de l'empereur que : « toute probabilité de leur rapatriement en Éthiopie a maintenant disparu pour toujours ${ }^{45}$; ce qui fut aussi l'avis des intellectuels jamaïcains. Rex Nettleford, qui avait cosigné le Rapport de 1960 sur le mouvement indiqua en 1970 que "la visite de Hailé Sellassié contribua au déclin de l'ardeur du désir d'un retour physique " (Nettleford, 2001 : 73). De même, Barry Chevannes ne releva aucune tentative de retour durant les années 1960 (Chevannes, 1998 : 30). Ainsi s'exprimait encore par la voix des universitaires la position gouvernementale qui essayait à tout prix d'étouffer les revendications au retour, mais en réalité toutes les arrivées en Éthiopie depuis la Jamaïque se firent après la visite de l'empereur en 1966. Deux mois après son départ le grand ghetto de Back-O-Wall fut rasé, jetant à la rue des centaines de personnes parmi lesquelles de nombreux rastafaris qui chantèrent alors : "Puisque nous sommes des squatters en Jamaïque/Renvoyeznous en Éthiopie/Là nous serons des citoyens "(Barrett, 1997 : 157). La visite d'Haile Sellassie ler et la répression qui s'ensuivit poussèrent des Jamaïcains à s'organiser pour quitter l'île et à s'installer en Éthiopie. Ils démontraient ainsi la façon dont ils s'étaient emparés de la recommandation du " rapport de 1960 " pour en subvertir les objectifs et matérialiser leur retour en Afrique. Les rastafaris ne comptèrent plus alors que sur eux-mêmes et commencèrent à préparer leur départ.

\section{La famille Baugh, première à quitter la Jamaïque}

En 1968 un petit groupe de quatre adultes et quatre enfants (la famille Baugh et un de leurs amis, Vivian Thompson) quitta la Jamaïque. Clifton Baugh naquit en 1925 à Swift River, à Portland où il grandit avant de migrer en ville. Inez Williams naquit en 1935 à St Ann's Bay, et elle resta longtemps en province avant de se diriger, elle aussi, vers la ville. Ils se rencontrèrent à Spanish Town où Clifton travaillait dans le bâtiment et Inez avait un emploi administratif. Elle avait été élevée dans I'Église méthodiste alors que Clifton était, dès ses vingt ans, familier du mouvement rastafari. Un de leur fils né en Éthiopie se rappelle la façon dont son père lui racontait l'origine de son intérêt pour l'Afrique :

"Mon père (...) commença à lire et entendre l'histoire de l'Éthiopie, il était inspiré par Marcus Garvey et le mouvement de retour en Afrique, c'est ça qui les a poussés à penser à bouger. (...) C'est là qu'ils eurent l'inspiration. (...) Mon père racontait qu'il avait I'habitude de voir ses amis, ils se retrouvaient après le travail et ils appelaient ça raisonnement, quand tu t'assieds et tu parles, tu vois ce que je veux dire? Alors un de ces soirs, après le travail ils se rencontrèrent et commencèrent à parler d'Afrique, c'est là que son désir de rentrer à la maison commença à monter, ils disaient, ils parlaient de rentrer à la maison, mais dans ce temps-là personne ne pouvait se permettre de payer le voyage et rentrer à la maison. Il y avait ces différents groupes, Black Star Liner, ramener les gens en bateau, mais c'était pas très concret $"^{46}$.

45 PRO, DO 200/170, Rapport confidentiel de J. D. Murray, haut commissionnaire britannique en Jamaïque à l'attention du secrétaire d'État pour le Commonwealth, 14/06/1966, p. 4.

46 Entretien avec D. Baugh, Addis-Abeba, Éthiopie, 04/10/2003. 
Le reasoning ou raisonnement était la forme de sociabilité la plus courante et diffuse parmi les rastafaris. II se tenait dans des yard, I'unité de résidence urbaine. La divinité de l'empereur y était construite sur un mode discursif et les pratiques culturelles propres aux rastafaris étaient transmises et partagées (Homiak, 1985). L'évocation de l'Afrique, de l'Éthiopie, de la " terre de leurs pères " était quotidiennement mise en scène à travers ces interactions sociales. La visite de Hailé Sellassié ler en 1966 fit basculer les Baugh des discours aux pratiques du retour. Inez, la mère, se souvient avec émotion de l'arrivée de l'empereur à Kingston. Elle était sur le tarmac avec, disait-elle, le plus grand drapeau de l'enthousiaste assemblée ${ }^{47}$. Leur fils confirma l'influence exercée par cette visite sur la dynamique de leur départ :

" Je me rappelle mon père qui disait que quand Sa Majesté est venue en Jamaïque, c'est là qu'ils ont été motivés, enfin, on ne va pas attendre plus longtemps si notre Père est déjà venu, et nous l'avons vu, il est vraiment temps pour nous de bouger. (...) Comme je vois les choses, si on ne s'occupe pas de notre propre déplacement, nous n'arriverons jamais à partir d'ici $\Perp^{48}$.

Se désignant eux-mêmes comme des "pionniers ", les Baugh vendirent leur maison et leurs biens pour financer leur voyage. Familiers des organisations et des yards des rastafaris, ils étaient militants pour le retour en Afrique sans pour autant être membres de I'EWF. En agissant de leur propre initiative, ils illustraient combien les organisations avaient en réalité du mal à encadrer tous les rastafaris. Les Baugh se heurtèrent à leurs familles et à leurs proches qui désapprouvaient cette initiative et reproduisaient l'imagerie populaire courante en Jamaïque qui identifiait l'Afrique à des valeurs négatives et primitives. Ils leur disaient qu'ils étaient " fous de quitter une terre et d'aller vers une autre terre toute vide où il y avait juste la famine et la guerre ${ }^{49}$. Mais leur décision était prise. La sœur d'Inez, attristée par ce départ, et avec peut-être l'espoir qu'elle les reverrait bientôt, leur confia sa fille. Sur une photo prise juste avant leur départ, ils semblaient calmes et sereins, les hommes, barbus, en costume et cravate avec une écharpe aux couleurs de l'Éthiopie et Inez en tailleur avec un bandeau dans les cheveux ${ }^{50}$. Après un long voyage, ils arrivèrent finalement en Éthiopie où ils retrouvèrent les quelques personnes déjà installées à Shashemene. Leur voyage signifia à la fois l'échec du gouvernement jamaïcain et le coup d'envoi des départs pour des Jamaïcains qui allaient se poursuivre avec régularité.

\footnotetext{
47 Entretien avec I. Baugh, Shashemene, Éthiopie, 09/11/2001.

48 Entretien avec D. Baugh, Addis-Abeba, Éthiopie, 04/10/2003.

49 Entretien avec D. Baugh, Addis-Abeba, Éthiopie, 04/10/2003.

50 DG, NLK, 08/02/1968, reprise dans AO, UCLA, 1968, 8 (7-8), p. 11.
} 
Photo 1 : La famille Baugh sur le départ pour l'Éthiopie :

Vivian Thompson porte Patricia Thompson, Inez Baugh porte Anne Marie Boyd, Clifton Baugh porte Jasmine Baugh et, au premier plan, Clifton Douglas

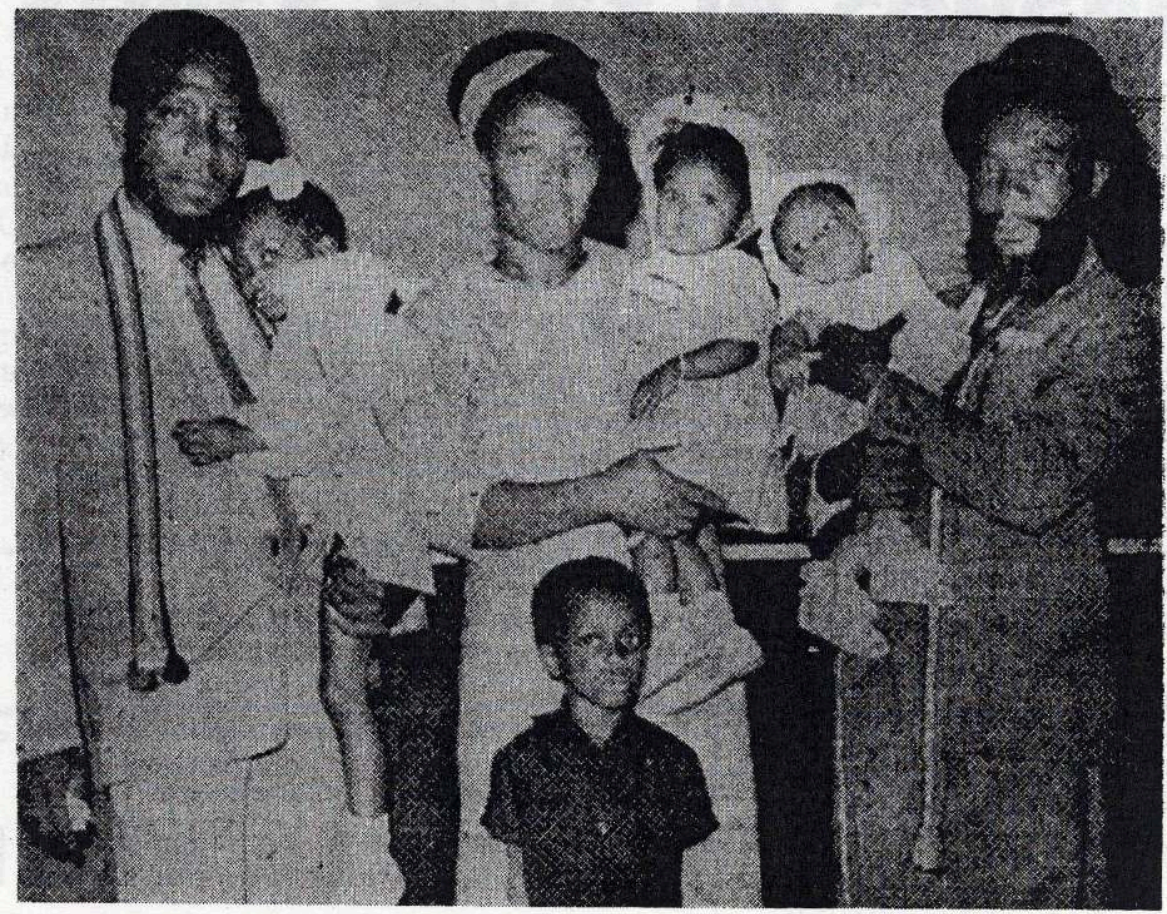

Source : African Opinion, UCLA Research Library, 1968, 8 (7-8), p. 11. (C) DR.

\section{Conclusion}

Le retour en Afrique représentait une menace pour la société jamaïcaine coloniale et post-coloniale. L'établissement d'une nation noire imaginée à travers le prisme du retour en Afrique se dressait comme une alternative à la domination britannique et à la nation jamaïcaine en construction. Les travailleurs engagés, notamment Sierra Léonais, à la fin du XIXe siècle, les vétérans de la Première Guerre mondiale, les garveyites après la chute de Garvey, réclamèrent le droit de rentrer en Afrique, à l'appui de leur statut de descendants d'esclaves africains. Les rastafaris recentrèrent ces revendications sur le départ en Éthiopie, soutenus par leur interprétation de la nature divine d'Hailé Sellassié sur qui se concentraient les imaginaires nationalistes et religieux. L'Ethiopian World Federation, à qui les terres de Shashemene avaient été confiées, fut d'abord réticente à intégrer les rastafaris principalement à cause de leurs croyances et de leurs pratiques. Pourtant, dans une société profondément structurée par les migrations internes et internationales, I'Afrique apparaissait durant les années 1950, comme une alternative possible de destination de migration, malgré une image très dévalorisée dans la société jamaïcaine. Le retour était alors un débat public en Jamaïque, entretenu par des voix fortes comme celles de Prince Emmanuel et Mortimer Planno. Le rapport de 1960 changea quelque peu 
le regard sur les rastafaris ainsi que la mission de 1961, première initiative du gouvernement pour étudier les possibilités migratoires vers I'Afrique. Avec I'indépendance en 1962 et la nouvelle légitimité du gouvernement, ces possibilités étaient enterrées. La visite de l'empereur en 1966 incita finalement les rastafaris à s'organiser sans attendre une aide gouvernementale, et le premier groupe de Jamaïcains partit en 1968 pour l'Éthiopie. Les relations entre le gouvernement jamaïcain et la population jamaïcaine, cristallisées autour du retour en Afrique, illustrent la tension sans cesse renouvelée entre une gestion étatique oscillant vers le refus, voire la négation des demandes, et une prudence dans l'exploration des possibilités d'un soutien à l'émigration vers I'Afrique. En outre, la critique sociale sous-tendue par les incessantes demandes au retour met en évidence les formes changeantes de l'appartenance nationale. La diversité des termes usités pour parler du déplacement vers l'Afrique - " déporter ", " retourner ", " émigrer ", " rapatrier " - témoigne des influences d'un contexte international qui s'est transformé entre les années 1920 et la fin des années 1960, ainsi que celles des représentations symboliques du retour. Le radicalisme associé au retour toujours entendu comme une responsabilité du gouvernement, semble s'être renforcé face à l'absence d'une politique claire. C'est finalement sans I'aide du gouvernement jamaïcain, voire contre le gouvernement, que des rastafaris, dès 1968, ont commencé leur " retour " vers l'Éthiopie.

\section{Références bibliographiques}

Agudelo Carlos, Boidin Capucine et Sansone Livio (Éds.) (2009) L'Atlantique noir. Une polyphonie de perspectives, Paris, IHEAL, $221 \mathrm{p}$.

Anteby-Yemini Lisa, Berthomière William et Sheffer Gabriel (Éds.) (2005) Les diasporas. 2000 ans d'histoire, Rennes, Presses Universitaires de Rennes, 497 p.

Barrett Leonard E. (1997 [1976]) The Rastafarians, Boston, Beacon Press, 306 p.

Bonacci Giulia (2013) The Ethiopian World Federation: a Pan-African Organization among the Rastafari in Jamaica, Caribbean Quarterly, pp. 73-95.

Bonacci Giulia (2010) Exodus ! L'histoire du retour des Rastafariens en Éthiopie, Paris, L'Harmattan, $536 \mathrm{p}$.

Campbell Horace (1994 [1987]) Rasta and resistance. From Marcus Garvey to Walter Rodney, Trenton (NJ), Africa World Press, 236 p.

Chevannes Barry (1998) New Approach to Rastafari, in Barry Chevannes Ed., Rastafari and other African-Caribbean Worldviews, La Haye, Institute of Social Studies, pp. 20-42.

Chevannes Barry (1994) Rastafari. Roots and Ideology, Syracuse, NY, Syracuse University Press, 298 p.

Chevannes Barry (1976) The Repairer of the Breach: Reverend Claudius Henry and Jamaican Society, in Francis Henry Ed., Ethnicity in the Americas, La Haye, Mouton, pp. 263-289.

Chivallon Christine (2004) La diaspora noire des Amériques. Expériences et théories à partir de la Caraïbe, Paris, CNRS Éditions, 258 p. 
Dufoix Stéphane (2011) La dispersion. Une histoire des usages du mot diaspora, Paris, Éditions Amsterdam, 573 p.

Hill Robert A. (2013) Our Man in Mona: A Conversation between Robert A. Hill and Annie Paul, Active Voice, [online] last checked on 04/10/2013. URL: http:// anniepaul.net/our-man-in-mona-an-interview-by-robert-a-hill-with-annie-paul/

Hill Robert A. (2001) Dread History. Leonard P. Howell and millenarian visions in the early Rastafarian religion, Chicago, Kingston, Research Associates School Times, Miguel Lorne Publishers, 64 p.

Hill Robert A. (Ed.) (1983) The Marcus Garvey and Universal Negro Improvement Association Papers, Vol. 1, Berkeley, Los Angeles, London, University of California Press, $579 \mathrm{p}$.

Homiak John (1985) The "ancient of days" seated Black: Eldership, Oral tradition and Ritual in Rastafari culture, Thèse de doctorat, anthropologie, Brandeis University, Waltham (Mass.), 537 p.

James Winston (1998) Holding Aloft the Banner of Ethiopia. Caribbean Radicalism in early Twentieth-century America, Londres, New York, Verso, 406 p.

Lee Hélène (1999) Le Premier Rasta, Paris, Flammarion, 367 p.

Lewis Rupert C. (1998) Marcus Garvey and the early Rastafarians: continuity and discontinuity, in N. Samuel Murrell, William David Spencer, Adrian Anthony McFarlane Eds., Chanting down Babylon. The Rastafari reader, Kingston, Ian Randle, pp. 145-158.

Mack Douglas (1999) From Babylon to Rastafari: Origin and history of the Rastafarian movement, Chicago, Research Associates SchoolTimes Publications, $157 \mathrm{p}$.

Majority Report of Mission to Africa (1961) (Presented to Prime Minister the Hon. Norman W. Manley), Kingston, Government Printer, 13 p.

Martin Tony (1986 [1976]) Race First. The Ideological and Organizational Struggles of Marcus Garvey and the Universal Negro Improvement Association, Dover (Mass.), The Majority Press, $421 \mathrm{p}$.

Minority Report of Mission to Africa (1961) (Presented to Prime Minister the Hon. Norman W. Manley), Kingston, Government Printer, 9 p.

Nettleford Rex (2001 [1970]) Mirror, Mirror. Identity, Race and Protest in Jamaica, Kingston, LMH Publishing, $256 \mathrm{p}$.

Patterson Orlando (1975 [1969]) The sociology of slavery; an analysis of the origins, development, and structure of Negro slave society in Jamaica, Londres, Associated University Press, 310 p.

Salewicz Chris (2000) Rude boy: once upon a time in Jamaica, Londres, V. Gollanz, 280 p.

Shepperson George (1962) Pan-Africanism and "Pan-Africanism": some historical notes, Phylon, 23 (4), pp.346-358.

Shuler Monica (1980) "Alas, Alas, Kongo". A social history of indentured African immigration into Jamaica, 1841-1965, Baltimore, Londres, The John Hopkins University Press, 186 p. 
Smith Michael Garfield, Augier Roy and Nettleford M. Rex (Eds.) (1960) The Rastafarian movement in Jamaica, Kingston, Institute of Social and Economic Research, $43 \mathrm{p}$.

Technical Mission to Africa (Nigeria, Ghana, Ethiopia) Summary Report, JanuaryMarch 1962 (2010) Caribbean Quarterly, Special Issue Rastafari. The Reports, pp. 77-107.

Van Dijk Frank Jan (1995) Sociological means: Colonial reactions to the radicalization of Rastafari in Jamaica, 1956-1959, New West Indian Guide, 69 (1-2), pp. 67-101.

Van Dijk Frank Jan (1993) Jahmaica. Rastafari and wider society, 1930-1990, Utrecht, ISOR, $482 \mathrm{p}$. 


\section{Giulia Bonacci}

\section{$\ldots$ La fabrique du retour en Afrique. Politiques et pratiques de I'appartenance en Jamaïque (1920-1968)}

Cet article se centre sur les Jamaïcains qui ont formulé leur désir de rentrer en Afrique à leur gouvernement, en envoyant des lettres, des pétitions et en créant plusieurs organisations. La diversité sociale des demandeurs est soulignée : travailleurs Sierra Léonais, descendants des esclaves africains dont les vétérans de la Première Guerre mondiale et les membres de I'Universal Negro Improvement Association de Marcus Garvey. L'auteure montre que le retour a joué un rôle particulier dans la naissance et le développement du mouvement rastafari jamaïcain, en catalysant leur critique sociale et politique. Face au poids social grandissant des rastafaris, le gouvernement soutint une mission en Afrique au début des années 1960 pour étudier les possibilités d'émigration. Mais c'est finalement l'échec de l'encadrement des demandes de retour par le gouvernement jamaïcain qui est signifié lorsque de petits groupes de rastafaris jamaïcains organisent eux-mêmes leur départ vers l'Éthiopie à la fin des années 1960.

\section{Constructing the Return to Africa. Politics and Practices of Belonging in Jamaica (1920-1968)}

This paper focuses on the Jamaicans who formulated to their government their desire to return to Africa thanks to letters, petitions and the creation of various organizations. The social diversity of the plaintiffs is underlined: workers from Sierra Leone, descendants of the African slaves including veterans of the First World War, and members of Marcus Garvey's Universal Negro Improvement Association. The return played a particular role in the birth and growth of the Rastafari movement, channelling their social and political critique. The growing social weight of Rastafari gave rise to public initiatives on behalf of the government in the early 1960s. Despite these initiatives, the failure of the government in responding to these queries is evidenced when small groups of Jamaican Rastafari organized themselves their own return to Ethiopia by the end of the 1960s.

\section{La fábrica del retorno en África. Políticas y prácticas de la pertenencia en Jamaica (1920-1968)}

Este artículo se interesa por los jamaicanos que expresaron a su Gobierno su deseo de volver a África a partir de cartas, peticiones y la creación de diversas organizaciones. Asimismo se subraya en el mismo la diversidad social de los solicitantes de retorno: trabajadores sierraleoneses, descendientes de los esclavos africanos incluidos los veteranos de la Primera Guerra Mundial y los miembros de la Universal Negro Improvement Association de Marcus Garvey. El retorno tuvo un papel particular en el nacimiento y desarrollo del movimiento rastafari jamaicano, como catalizador a la vez de su crítica social y política. El peso social en aumento del movimiento rastafari suscito iniciativas públicas gubernamentales a principios de los años 1960 del siglo pasado. A pesar de estas iniciativas, el fracaso por parte del gobierno jamaicano en la gestión de las peticiones de retorno se pone de manifiesto al constatar que pequeños grupos de rastafaris jamaicanos organizaron por cuenta propia un retorno a Etiopía a finales de la misma década. 